\title{
Mechanism and Kinematics for Flapping Wing Micro Air Vehicles Maneuvering Based on Bilateral Wings
}

\author{
Rui Zhang $\mathbb{D}^{1},{ }^{1}$ Haiyang Zhang, ${ }^{1}$ Lichao Xu, ${ }^{1}$ Peng Xie, ${ }^{2}$ Jing Wu, ${ }^{3}$ and Chao Wang ${ }^{3}$ \\ ${ }^{1}$ School of Traffic Engineering, Huaiyin Institute of Technology, Huai'an 223003, China \\ ${ }^{2}$ Sino-German College of Intelligent Manufacturing, Shenzhen Technology University, Shenzhen 518118, China \\ ${ }^{3}$ School of Mechanical Engineering, Dongguan University of Technology, Dongguan 523808, China
}

Correspondence should be addressed to Rui Zhang; zhangr_hyit@qq.com

Received 8 October 2021; Revised 28 December 2021; Accepted 6 January 2022; Published 2 February 2022

Academic Editor: Giulio Avanzini

Copyright () 2022 Rui Zhang et al. This is an open access article distributed under the Creative Commons Attribution License, which permits unrestricted use, distribution, and reproduction in any medium, provided the original work is properly cited.

With the development and application of a bionic flapping micro aircraft vehicle (FMAV), further requirements for its high maneuvering flight characteristics have been put forward. A controlling tail cannot meet the requirements of the maneuvering flight of insect-sized FMAVs, and it has become necessary to find new mechanisms for high maneuvering flight. In this study, two flapping mechanisms with movable hinges based on a double crank rocker mechanism (DCRM) and a single crank slider mechanism (SCSM) are proposed. The instantaneous flapping angles, their extremum and means, and the flapping amplitudes of the two mechanisms are studied for various hinge offsets. A comparison between these two mechanisms indicates that the mechanism based on SCSM is more appropriate in providing adjustable flapping amplitudes for lateral wings. Finally, a dragonfly-like prototype assembled using a carbon fiber plate with adjustable wings is designed and manufactured based on the SCSM. Flight tests are conducted to show that the rack and pinion mechanism can accomplish the movement of hinges neatly, and the proposed flapping mechanism makes left turns and right turns effectively while continuing to fly forward. The prototype is good in terms of crashworthiness and the replacement of components, and it offers an easy and reliable technique for the maneuvering flight of FMAVs.

\section{Introduction}

Compared to micro air vehicles (MAVs) with fixed-wings or rotors, micro air vehicles with flapping wings (FMAVs) based on bionics have more advantages, such as a low flapping frequency, good abilities for camouflage, and excellent aerodynamic performance at low Reynolds numbers. FMAVs have a wide range of application prospects in the military and civilian fields, such as in reconnaissance, wiretaps, small space searches after a disaster, and tracking shots. This has put forward higher requirements for the maneuverability of FMAVs. In fact, the high maneuverability of flapping wings has been a focus of many studies involving research about the high lift mechanics and the general rules of the aerodynamic performance of flapping wings $[1,2]$, such as studies on the maneuvering flights of dragonflies by Alexander [3, 4], Wang et al. [5], and Zhang et al. [6]; studies of fruit flies by Fry et al. [7], Ramamurti and Sandberg [8],
Ristroph et al. [9], Vogel [10], Muijres [11], and Meng and Sun [12]; and studies of hummingbirds by Nan et al. [13, 14], Fei et al. [15], and Timmermans et al. [16].

The turning performance is one of the most basic performances in maneuvering flight. The wind tunnel study of tied dragonflies by Alexander [3] showed that there were two different turn modes, namely, the conventional turn mode and the yaw turn mode. In the first mode, the asymmetric flapping amplitudes and the occasional asymmetric angles of the attack of bilateral wings made the dragonflies roll into a bank, which was similar to the turning of airplanes. In another mode, the drags on the inner wings up-stroke and the outer wings down-stroke made the dragonflies turn, much like pivoting a boat. The conventional turn mode was also found in the turning observations of fruit flies by Muijres [11]. It is known that both dragonflies and fruit flies use left-right asymmetries in the wing stroke amplitude to produce aerodynamic moments to turn $[4,10]$. For fruit 
flies, it is the inertia force and not the viscosity force that plays a dominant role [7], while for small flying insects, the effect of the inertia force is not significant [17]. A numerical study on the turning flight of fruit flies by Ramamurti and Sandberg [8] showed that the thrust and lift generated the turning moment together, and the lateral force produced the righting moment separately. The lateral force is related to the left-right asymmetries in the wing angle of attack, and this force can also be generated by changing the relative turnover times of lateral wings [9]. In other words, dragonflies or insects with similar sizes obtain their maneuverability when turning by changing the flapping parameters of their lateral wings, such as their flapping amplitudes [3-6, 10], angles of attack [3, 9, 18], and or flapping frequencies [19-21], rather than any parameters related to their tails [22-24].

There have been some FMAVs with adjustable mechanisms for turning flight that have been developed based on the preliminary understanding of flapping turning flight [23, 25-27]. However, the flapping parameter regulating mechanisms of these FMAVs are too complex, and the FMAVs usually have a large turn radius and poor maneuverability. In fact, the turn radius and the maximum turn acceleration for real dragonflies are $0.08 \mathrm{~m} \mathrm{[28]} \mathrm{and} 9 \mathrm{~g}$ [29], respectively, surpassing most of the existing FMAVs. High maneuverability with a simple flapping mechanism for an FMAV is the key to practical applications. In this study, a flapping mechanism that allows the left and right flapping amplitudes to adjust simultaneously is proposed based on the basic flapping mechanisms, namely, the double crank rocker mechanism (DCRM) and the single crank slider mechanism (SCSM), and the performances of these two mechanisms are studied in detail. Finally, an FMAV with adjustable flapping wings is manufactured and tested.

\section{Materials and Methods}

2.1. Hinge Movement. The flapping mechanism is the most important part of an FMAV. The DCRM and the SCSM are typical and common symmetric flapping mechanisms used for FMAVs [30] (Figures 1(a) and 1(c)). It is assumed that the flapping hinges of these two mechanisms are not fixed but rather allowed to move horizontally, as shown in Figure 1. The common hinge of two flapping rods of the DCRM is movable along the sliding chute in the frame (Figure 1(c)), and the two hinges of the SCSM are synchronously movable along the sliding chute (Figure $1(\mathrm{~d})$ ). The instantaneous flapping angles between the left-right flapping bars and the horizontal plane are marked as $\phi_{L}$ and $\phi_{R}$. Because the flapping hinges move, $\phi_{L}$ and $\phi_{R}$ are not equal during the flapping period. Hence, an asymmetry in the lateral flapping amplitude is obtained.

2.2. Analysis of Kinematics. A diagram of the kinematics analyses of the DCRM and the SCRM is given in Figure 2. In the DCRM, the origin of the coordinates $O$ is located at the contact point of two gears, where the right gear is the driver. The radii of two gears are equal, and they are both denoted by $r$. The $x$-axis is rightward, and the $y$-axis is upward. The two gears rotate around points $A_{L}$ and $A_{R}$. The location of the hinge after its movement is denoted by $C$. The length of a rocker is marked as $l_{2}$. The angle between the crank $A_{R} B_{R}$ and the $x$-axis is denoted by $\theta$, where $\theta$ is positive when the crank rotates anticlockwise. Cranks $A_{L} B_{L}$ and $A_{R} B_{R}$ are installed symmetrically because of the symmetry required in forward flight. At the initial moment, crank $A_{R} B_{R}$ is coincident with the $x$-axis. The angle between the left connecting rod $B_{L} D_{L}$ and the left flapping rod $C D_{L}$ is marked as $\varphi_{L}$. Similarly, the angle between $B_{R} D_{R}$ and $C D_{R}$ is marked as $\varphi_{R}$.

In the SCSM, the origin of coordinates $O$ is located at the rotation center of the crank. The distances from the lateral hinges $(C$ and $D)$ at the equilibrium position $(\tau=0)$ to the $y$-axis are equal, and these distances are marked as $l_{2}$. Hence, the distance between the two hinges is $2 l_{2}$ all the time during their movements. The rotational angle of crank $O A$ is denoted by $\theta$, and in the beginning, crank $O A$ is parallel to the $x$-axis and starts with anticlockwise rotation. The angle between the connecting rod $A B$ and the $y$-axis is marked as $\varphi$, and it is positive when it is on the right side of the $y$-axis.

The lengths of the cranks and the connecting rods are denoted by $r_{0}$ and $l_{1}$, respectively. $l_{0}$ is the distance from the origin of the coordinates $O$ to the sliding chute that the hinges move along. $\Delta l$ is the distance that the hinges move from equilibrium position ( $y$-axis), and it is positive to the right side of the $y$-axis. Obviously, $\Delta l=0$ when the hinges are at the equilibrium position, and at this moment, the lateral wings flap symmetrically. $\phi_{L}$ is positive when the flapping bar rotates clockwise, and $\phi_{R}$ is the opposite. It is obvious that the relationships of $\theta$ versus $\phi_{L}$ and $\phi_{R}$ will reveal the effect of the offset $\Delta l$ on the instantaneous flapping angles.

In the DCRM,

$$
\overrightarrow{A_{R} D_{R}}=\overrightarrow{A_{R} B_{R}}+\overrightarrow{B_{R} D_{R}}
$$

where

$$
\begin{gathered}
\overrightarrow{A_{R} D_{R}}=\left(l_{2} \cos \varphi_{R}-r+\Delta l, l_{0}+l_{2} \sin \varphi_{R}\right), \\
\overrightarrow{A_{R} B_{R}}=\left(r_{0} \cos \theta, r_{0} \sin \theta\right), \\
\overrightarrow{B_{R} D_{R}}=\left(l_{1} \cos \left(\varphi_{R}+\phi_{R}\right), l_{1} \sin \left(\varphi_{R}+\phi_{R}\right)\right), \\
\phi_{R}=\arccos \frac{l_{2}^{2}+l_{1}^{2}-{\overrightarrow{C B_{R}}}_{2}^{2}}{2 l_{2} l_{1}}, \\
\overrightarrow{C B_{R}}=\left(r+r_{0} \cos \theta-\Delta l,-\left(l_{0}-r_{0} \sin \theta\right)\right) .
\end{gathered}
$$

Substituting Equations (1) to (6) into Equation (1), we obtain

$$
l_{2} \cos \varphi_{R}-r+\Delta l=r_{0} \cos \theta+l_{1} \cos \left(\varphi_{R}+\phi_{R}\right) .
$$




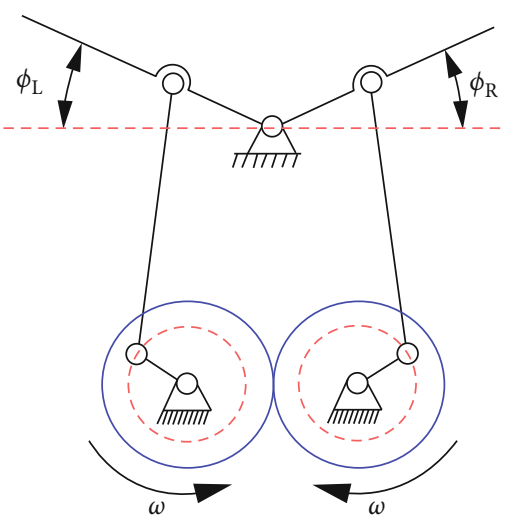

(a) Fixed hinge in DCRM

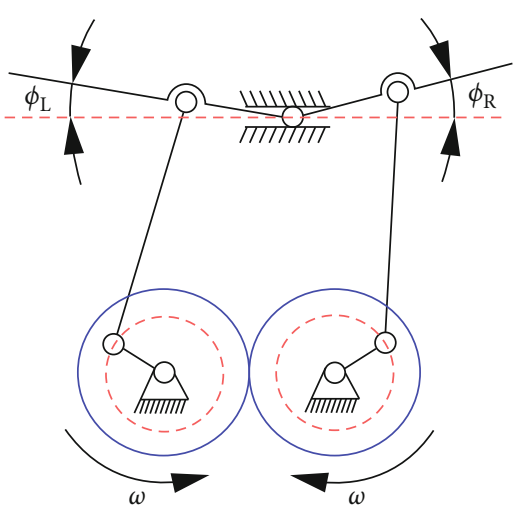

(b) Movable hinge in DCRM

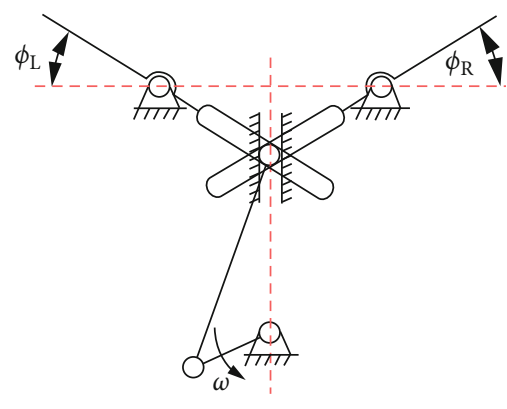

(c) Fixed hinges in SCSM

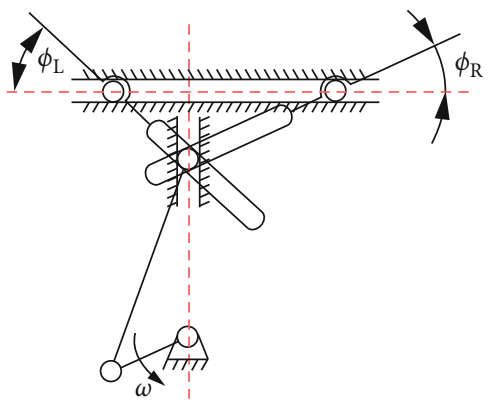

(d) Movable hinges in SCSM

Figure 1: Diagram of hinge movement in DCRM and SCSM.

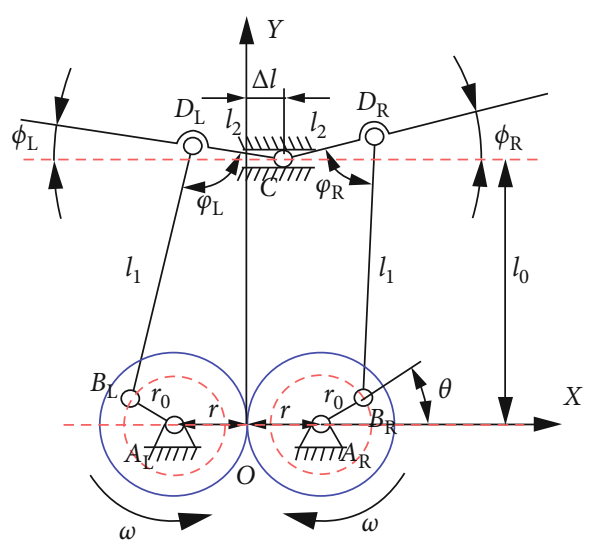

(a) Kinematic analysis of DCRM

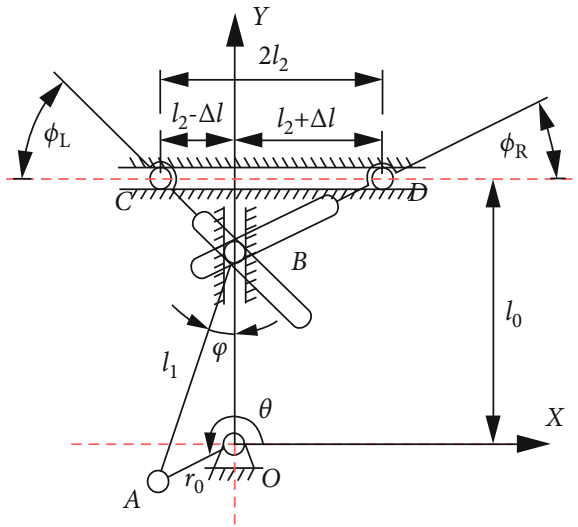

(b) Kinematic analysis of SCRM

FIGURE 2: Diagram of kinematic analysis.

TABLE 1: Lengths of all parts in the DCRM and the SCSM.

\begin{tabular}{lccccc}
\hline & $r(\mathrm{~mm})$ & $r_{0}(\mathrm{~mm})$ & $l_{0}(\mathrm{~mm})$ & $l_{1}(\mathrm{~mm})$ & $l_{2}(\mathrm{~mm})$ \\
\hline DCRM & 10.0 & 7.1 & 31.3 & 32.5 & 12.5 \\
SCSM & - & 10.0 & 31.3 & 28.3 & 13.0 \\
\hline
\end{tabular}

Similarly, we obtain the relationship between $\theta$ and $\phi_{L}$ as follows:

$$
r-l_{2} \cos \varphi_{L}+\Delta l=-r_{0} \cos \theta-l_{1} \cos \left(\varphi_{L}+\phi_{L}\right) \text {, }
$$

where

$$
\begin{gathered}
\phi_{L}=\arccos \frac{l_{2}^{2}+l_{1}^{2}-{\overrightarrow{C B_{L}}}^{2}}{2 l_{2} l_{1}}, \\
\overrightarrow{C B_{L}}=\left(-r-r_{0} \cos \theta-\Delta l, r_{0} \sin \theta-l_{0}\right) .
\end{gathered}
$$

Similarly, in the SCSM, we obtain

$$
\overrightarrow{\mathrm{OD}}=\overrightarrow{\mathrm{OB}}+\overrightarrow{\mathrm{BD}}
$$




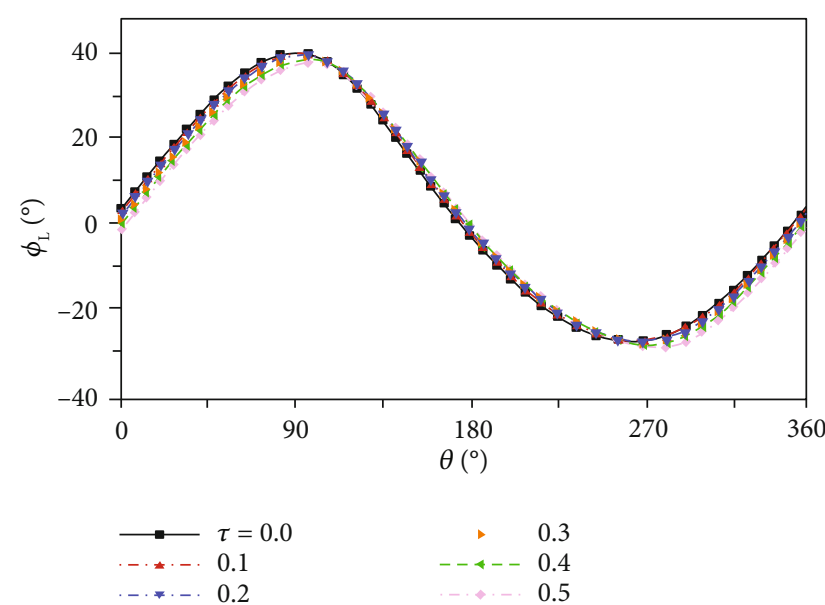

(a) $\phi_{L}$ vs. $\theta$

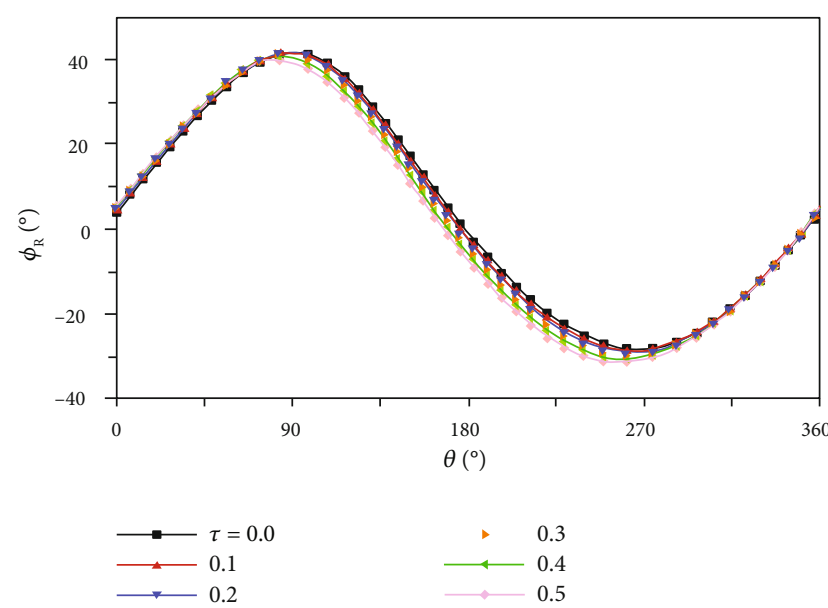

(b) $\phi_{R}$ vs. $\theta$

Figure 3: Relationships of $\phi_{L}$ and $\phi_{R}$ versus $\theta$ (DCRM).

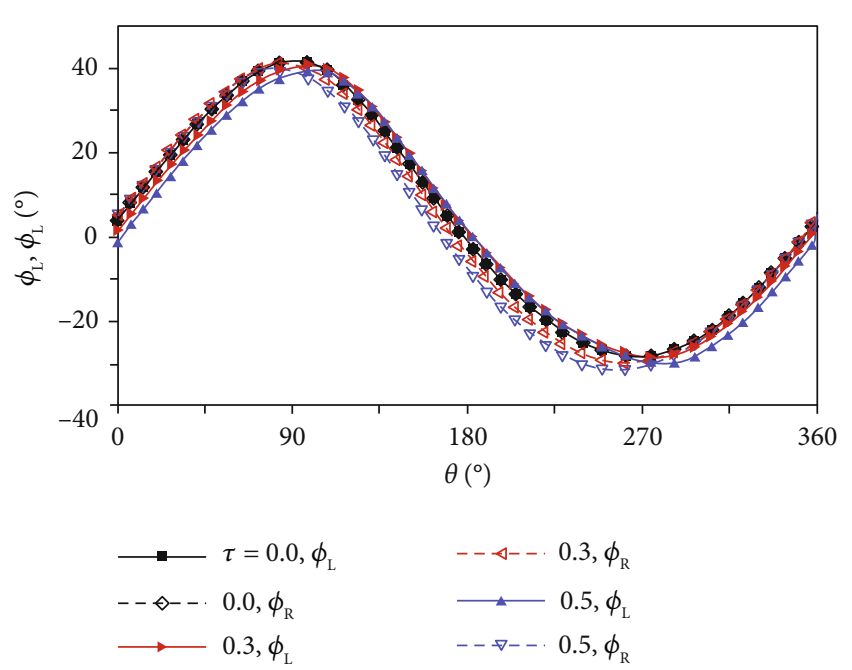

Figure 4: Comparison between $\phi_{L}$ and $\phi_{R}$ at various $\tau$ (DCRM).

where

$$
\begin{gathered}
\overrightarrow{\mathrm{OD}}=\left(l_{2}+\Delta l, l_{0}\right), \\
\overrightarrow{\mathrm{OB}}=\left(r_{0} \cos \alpha-l_{1} \sin \phi, r_{0} \sin \phi+l_{1} \cos \phi\right), \\
\overrightarrow{\mathrm{BD}}=\left(l_{2}+\Delta l,\left(l_{2}+\Delta l\right) \tan \varphi_{\mathrm{R}}\right) .
\end{gathered}
$$

From Equations (10) to (13), we obtain the relationship of $\phi_{L}$ and $\phi_{R}$ versus $\theta$ as follows:

$$
\begin{gathered}
\varphi_{L}=\arctan \frac{l_{0}-r_{0} \sin \theta-l_{1} \cos \left(\arcsin \left(r_{0} \cos \theta / l_{1}\right)\right)}{l_{2}-\Delta l}, \\
\varphi_{R}=\arctan \frac{l_{0}-r_{0} \sin \theta-l_{1} \cos \left(\arcsin \left(r_{0} \cos \theta / l_{1}\right)\right)}{l_{2}+\Delta l} .
\end{gathered}
$$

In addition to the instantaneous flapping angles, it is necessary to understand the effects of the hinge movement on the lateral flapping amplitude and the mean flapping angle. The flapping amplitude and the mean flapping angle are defined by $\phi_{0}=\left(\phi_{\max }-\phi_{\min }\right) / 2$ and $\bar{\phi}=\left(\phi_{\max }+\phi_{\min }\right) / 2$, respectively, where $\phi_{\max }$ and $\phi_{\min }$ are the maximum and the minimum of the instantaneous flapping angle. $\phi_{0}$ shows the magnitude of the flapping angle intuitively, and $\bar{\phi}$ reflects the global offset of the flapping angle. $\bar{\phi}$ has a certain effect on the lateral stability, and a large change of $\bar{\phi}$ is not desired when the hinges move.

For comparison, the lengths of all parts in the DCRM and the SCSM are selected in such a way that the maximum, minimum, amplitude, and mean of the instantaneous flapping angles in the DCRM are as close as possible to those in the SCSM at $\Delta l=0$, and the selected values are as shown in Table 1. To analyze the effect of the hinge movements of the DCRM and the SCSM, two dimensionless parameters named the relative hinge offset $\tau$ and the relative amplitude increment $\eta$ are introduced and defined as $\tau=\Delta l / r_{0}$ and $\eta=\left(\phi_{0}-\phi_{0 \mid \tau=0.0}\right) / \phi_{0 \mid \tau=0.0}$, respectively, where $\phi_{0 \mid \tau=0.0}$ is the flapping amplitude at the equilibrium position.

\section{Results and Discussion}

3.1. Instantaneous Flapping Angles. The relationships of $\phi_{L}$ and $\phi_{R}$ versus $\theta$ in the DCRM for different $\tau$ are shown in Figure 3. It is illustrated that the hinge movement affects both $\phi_{L}$ and $\phi_{R}$. When $\tau=0.0$, both $\phi_{L}$ and $\phi_{R}$ have their maximum and minimum values at $\theta=90^{\circ}$ and $\theta=270^{\circ}$, respectively. As $\tau$ increases, their maximum and minimum values decrease, and the curves of $\phi_{L}$ shift to the right side while those of $\phi_{R}$ do the opposite. It is also seen from Figure 3 that $\tau$ has a relatively larger effect on $\phi_{R}$ when $\theta$ is in the range of $90^{\circ}$ to $270^{\circ}$.

The comparisons between $\phi_{L}$ and $\phi_{R}$ for $\tau=0.0,0.3$, and 0.5 are shown in Figure 4. When $\tau=0.0$, the courses of $\phi_{L}$ 


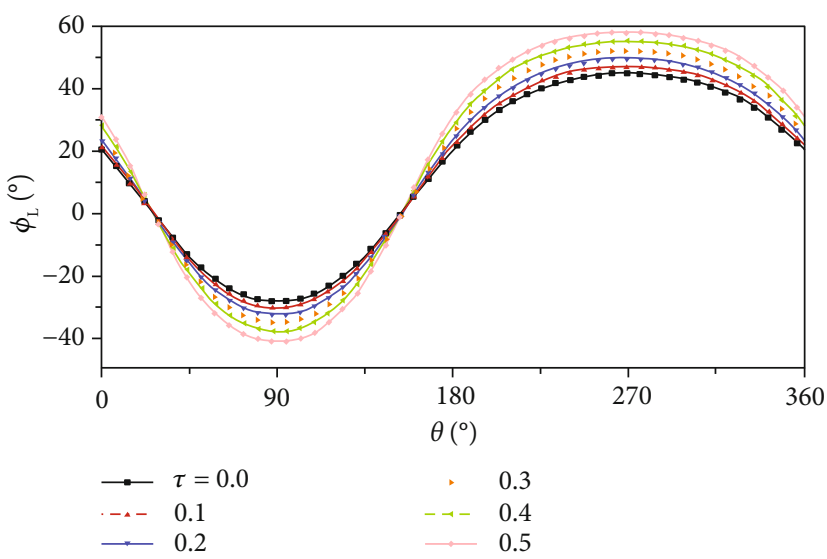

(a) $\phi_{L}$ vs. $\theta$

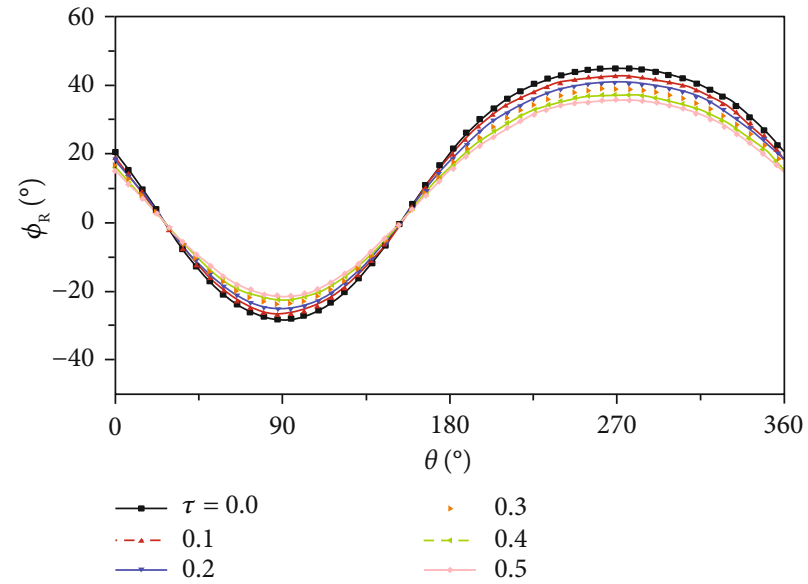

(b) $\phi_{R}$ vs. $\theta$

FIGURE 5: Relationships of $\phi_{L}$ and $\phi_{R}$ versus $\theta$ (SCSM).

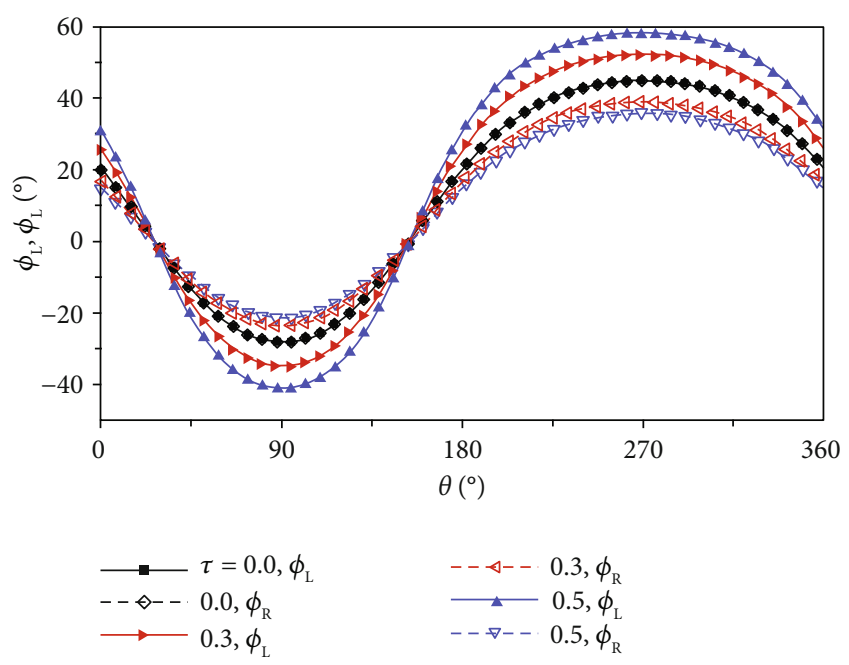

Figure 6: Comparison between $\phi_{L}$ and $\phi_{R}$ at various $\tau$ (SCSM).

and $\phi_{R}$ coincide for all $\theta$, which ensures a forward flight. As the hinge moves $(\tau=0.3), \phi_{L}$ and $\phi_{R}$ are no longer equal, and the symmetric flapping is broken. When $\tau=0.5$, the asymmetry becomes more obvious. It is important to note that $\phi_{L}$ is larger than $\phi_{R}$ when $\theta$ is in the range of $90^{\circ}-270^{\circ}$ which results in a right-side turning flight, and $\phi_{L}$ is smaller than $\phi_{R}$ in the other angle ranges, resulting in a left-side turning flight. This means that as the hinge moves, there is half a cycle where an FMAV with a DCRM will turn left and then turn right for the rest of the cycle. It is not good for the control of an FMAV if the movement of the hinge keeps the flapping angle on one side larger than that on the other side.

The relationships of $\phi_{L}$ and $\phi_{R}$ versus $\theta$ in the SCSM at different $\tau$ are presented in Figure 5. Conversely, when $\tau=$ $0.0, \phi_{L}$ and $\phi_{R}$ have their minimum and maximum at $\theta=$ $90^{\circ}$ and $\theta=270^{\circ}$, respectively. It is worth noting that the corresponding angles where $\phi_{L}$ and $\phi_{R}$ have their maximum and minimum in the SCSM are unchanged for different values of $\tau$, and the periods of time of the up-stroke and down-stroke are equal. That is to say, the moments when the flapping bars move to the peak and the bottom only depend on the crank. The offsets of the hinges change nothing but the extremums of $\phi_{L}$ and $\phi_{R}$. It is desired that as $\tau$ increases, the maximum of $\phi_{L}$ increases and its minimum decreases so that the range of $\phi_{L}$ becomes larger, or the maximum of $\phi_{R}$ decreases, and its maximum increases to make the range of $\phi_{R}$ smaller.

The comparison between $\phi_{L}$ and $\phi_{R}$ for $\tau=0.0,0.3$, and 0.5 are presented in Figure 6. When $\tau=0.0, \phi_{L}$ and $\phi_{R}$ are equal for all $\theta$ which ensures a stable forward flight. When $\tau=0.3, \phi_{L}$ increases, and $\phi_{R}$ decreases which makes an asymmetric flapping. When $\tau=0.5$, the differences in extremums between $\phi_{L}$ and $\phi_{R}$ are much larger, and the asymmetry becomes more obvious. With this value of $\tau$, the movement of two hinges makes an asymmetric flapping with difference lateral flapping amplitudes of $\phi_{0 L}$ larger and $\phi_{0 R}$ smaller. Larger difference between $\phi_{0 L}$ and $\phi_{0 R}$ will supply larger lateral force which is very important to the turn maneuverability $[3,4]$. It is known that a flapping flight will turn to the right as if $\phi_{0 L}$ is larger than $\phi_{0 R}[6]$; therefore, the movement of the hinges to right side will make FMAV turn to the same side. Since SCSM itself is symmetric, this does the same to the left side. In a word, an FMAV with such SCSM will turn to the same side as the hinges move to.

3.2. Comparison of Flapping Angles. The maximums, minimums, means, and amplitudes of $\phi_{L}$ and $\phi_{R}$ at various $\tau$ are presented in Table 2 , and the increment in amplitude $\eta$ is also given in the table.

In the DCRM, there is only a $2.15^{\circ}$ difference in the maximum of $\phi_{L}$ (from $41.57^{\circ}$ to $39.42^{\circ}$ ), a $1.7^{\circ}$ difference in the minimum, a $1.93^{\circ}$ difference in the mean, and a $0.22^{\circ}$ difference in the amplitude as $\tau$ increases from 0 to 0.5 , and the increment of amplitude $\eta$ is only $-0.63 \%$. This indicates that the influence of the hinge movement on $\phi_{0 L}$ is small. It is also seen from the table that the effect of the hinge 
TABLe 2: Maximums, minimums, means, and amplitudes of $\phi_{L}$ and $\phi_{R}$.

\begin{tabular}{ccccccccccccc}
\hline & $\tau$ & $\phi_{L \max }\left({ }^{\circ}\right)$ & $\phi_{L \min }\left({ }^{\circ}\right)$ & $\bar{\phi}_{L}\left(^{\circ}\right)$ & $\phi_{0 L}\left(^{\circ}\right)$ & $\eta$ & $\phi_{R \max }\left({ }^{\circ}\right)$ & $\phi_{R \min }\left({ }^{\circ}\right)$ & $\bar{\phi}_{R}\left({ }^{\circ}\right)$ & $\phi_{0 R}\left({ }^{\circ}\right)$ & $\eta$ \\
\hline \multirow{6}{*}{ DCRM } & 0.0 & 41.57 & -28.27 & 6.65 & 34.92 & 0 & 41.57 & -28.27 & 6.65 & 34.92 & 0 \\
& 0.1 & 41.40 & -28.16 & 6.62 & 34.78 & $-0.40 \%$ & 41.50 & -28.56 & 6.52 & 35.08 & $0.46 \%$ \\
& 0.2 & 41.09 & -28.26 & 6.41 & 34.68 & $-0.69 \%$ & 41.46 & -29.05 & 6.21 & 35.25 & $0.95 \%$ \\
& 0.3 & 40.65 & -28.58 & 6.04 & 34.62 & $-0.86 \%$ & 41.16 & -29.71 & 5.73 & 35.43 & $1.46 \%$ \\
& 0.4 & 40.09 & -29.14 & 5.48 & 34.62 & $-0.86 \%$ & 40.67 & -30.54 & 5.07 & 35.61 & $1.98 \%$ \\
& 0.5 & 39.42 & -29.97 & 4.72 & 34.70 & $-0.63 \%$ & 39.99 & -31.53 & 4.23 & 35.77 & $2.43 \%$ \\
& 0.0 & 45.00 & -28.29 & 8.35 & 36.65 & 0 & 45.00 & -28.29 & 8.35 & 36.65 & 0 \\
& 0.1 & 47.29 & -30.25 & 8.52 & 38.77 & $5.78 \%$ & 42.88 & -26.56 & 8.16 & 34.72 & $-5.27 \%$ \\
SCSM & 0.2 & 49.76 & -32.46 & 8.65 & 41.11 & $12.78 \%$ & 40.91 & -25.01 & 7.95 & 32.96 & $-10.07 \%$ \\
& 0.3 & 52.43 & -34.99 & 8.72 & 43.71 & $19.26 \%$ & 39.09 & -23.62 & 7.73 & 31.36 & $-14.43 \%$ \\
& 0.4 & 55.30 & -37.87 & 8.72 & 46.59 & $27.12 \%$ & 37.40 & -22.38 & 7.51 & 29.89 & $-18.44 \%$ \\
& 0.5 & 58.39 & -41.18 & 8.61 & 49.79 & $35.85 \%$ & 35.84 & -21.25 & 7.30 & 28.54 & $-22.13 \%$ \\
\hline
\end{tabular}

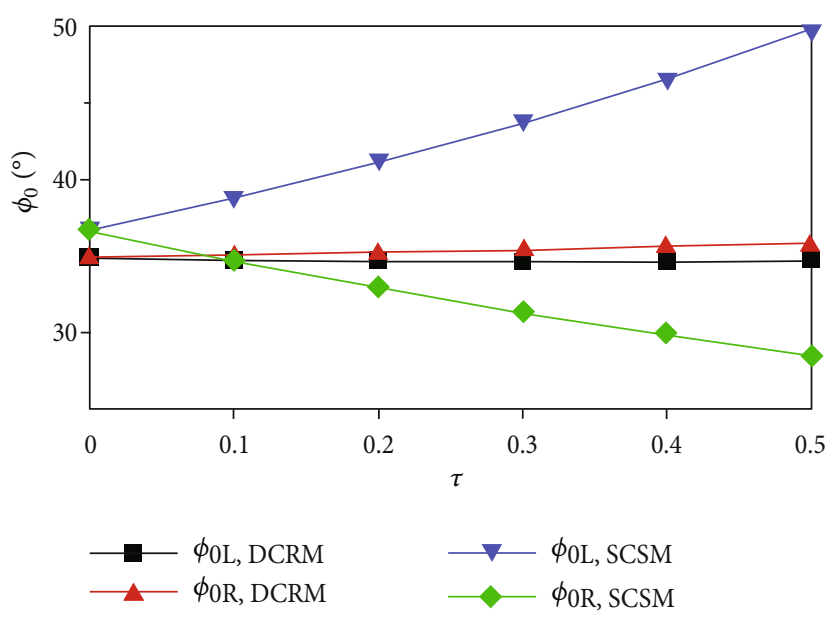

(a) $\phi_{0}$ vs. $\tau$

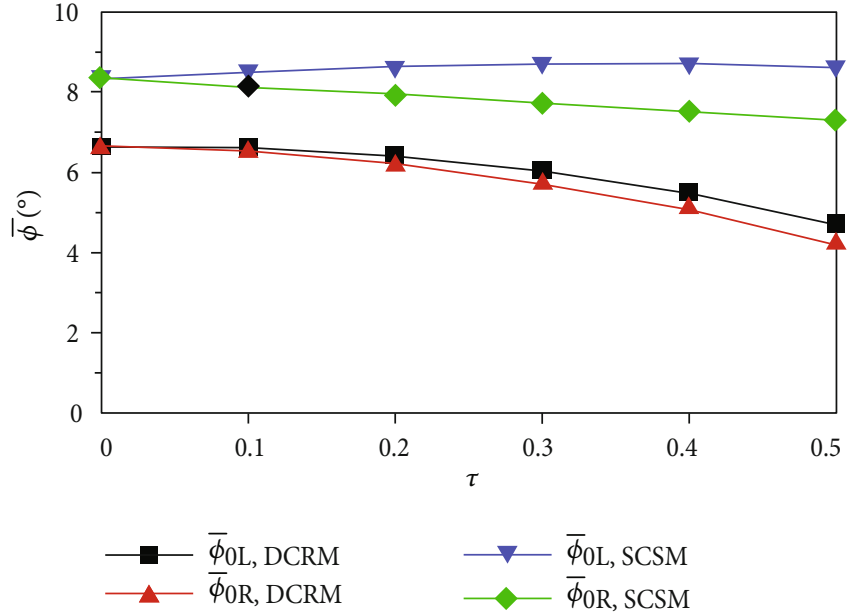

(b) $\bar{\phi}$ vs. $\tau$

FIgure 7: Comparison of $\phi_{0}$ and $\bar{\phi}$ at various $\tau$ for the DCRM and the SCSM.

movement on $\phi_{R}$ is similar, and its $\eta$ is less than $3 \%$. The overall data show that the movement of the hinge cannot provide enough influence for the FMAV to have a turning flight.

In the SCSM, however, the variations in these values are much larger as $\tau$ changes from 0 to 0.5 . The maximum of $\phi_{L}$ increases by $13.69^{\circ}$ from $45.00^{\circ}$ to $58.69^{\circ}$, and the minimum decreases by $12.89^{\circ}$ from $-28.29^{\circ}$ to $-41.18^{\circ}$. Additionally, $\phi_{0 L}$ increases by $13.14^{\circ}$ from $36.65^{\circ}$ to $49.79^{\circ}$, and the increment of amplitude $\eta$ is as high as $35.85 \%$. For $\phi_{R}$, similar influences are found, although they are relatively smaller, and the increment of amplitude is over 20 percent $(\eta=-22.13 \%)$. The overall data show that the movement of the hinges in the SCSM can provide enough influence for the FMAV to have a turning flight, and this can be an effective control mechanism for the turning flight of an FMAV.

To see the relationships of $\phi_{0 L}, \phi_{\mathrm{oR}}$, and $\bar{\phi}$ versus $\tau$ in the DCRM and the SCSM more intuitively, the curves drawn based on the data in Table 2 are shown in Figure 7. It is displayed clearly that $\phi_{0 L}$ and $\phi_{0 R}$ in the SCSM obviously change with $\tau$, and in contrast, $\phi_{O L}$ and $\phi_{0 R}$ in the DCRM are almost unchanged. It is worth mentioning that the changes of $\phi_{0 L}$ and $\phi_{0 R}$ in the SCSM are almost linearly dependent on $\tau$. In the DCRM, both $\bar{\phi}_{L}$ and $\bar{\phi}_{R}$ decrease with $\tau$, and the decrease becomes faster at large $\tau$, which is unfavorable for the lateral stability. $\bar{\phi}_{L}$ and $\bar{\phi}_{R}$ in the SCSM change more slowly than those in the DCRM. Hence, the SCSM is a more suitable mechanism for the turning flight of an FMAV.

3.3. FMAV Assembled with a Carbon Fiber Plate. A dragonfly FMAV assembled by carbon fiber plate with adjustable flapping wings based on the SCSM is shown in Figure 8 . The FMAV has better crashworthiness and replaceability of components than that made of acrylonitrile butadiene styrene. Two pairs of wings are driven by a motor driver. The driver drives the back crankshaft 


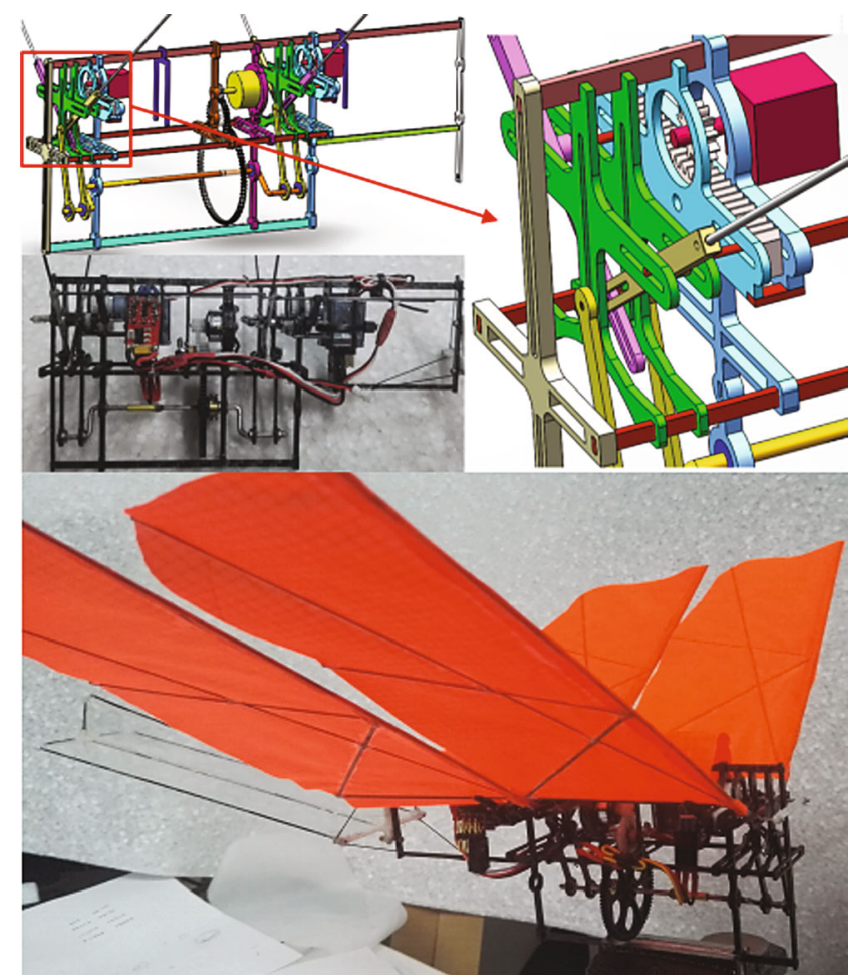

FIGURE 8: Three-dimensional FMAV model based on the SCSM.

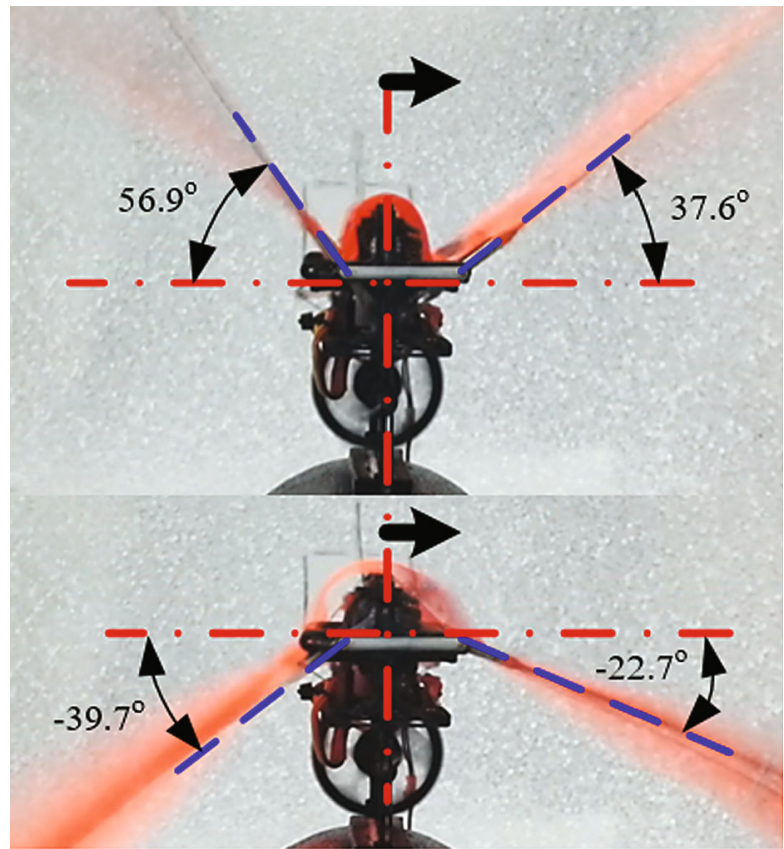

(a) $\tau=0.5$

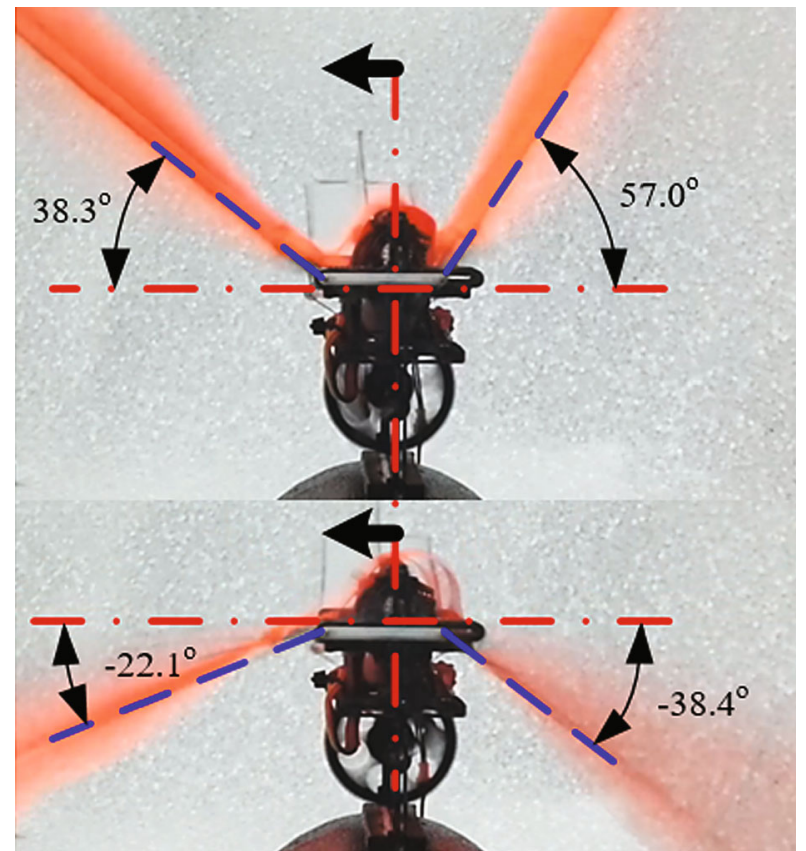

(b) $\tau=-0.5$

FIGURE 9: Maximum and minimum flapping angles at $\tau= \pm 0.5$.

directly after being driven by a gear reducer, and the front crankshaft is driven indirectly by a coupling. The flapping axes are fixed on the racks, which are driven by fore and after servos synchronously. The racks can move in the sliding chutes freely, driving the hinges to move and control the FMAV turning flight. 


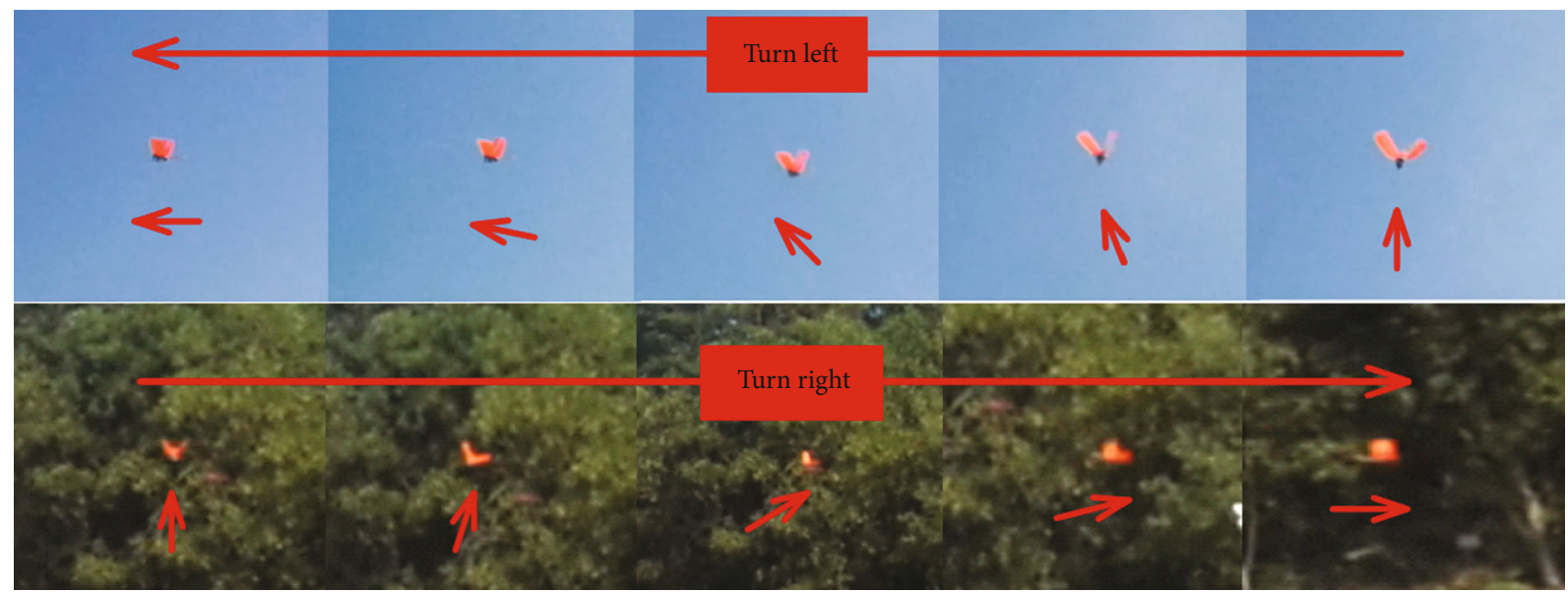

Figure 10: Test of turn flight.

If a control signal is not received, the servos do not drive the racks, and the racks are in the middle of the sliding chutes (equilibrium position). If a signal for turning right is received, the servos drive the racks to the right so that the left flapping amplitude is larger than the right flapping amplitude, resulting in a right turn for the FMAV. Similarly, the servos make the FMAV turn left when a left turn signal is received.

The prototype is tested on an experiment platform. The smoothness of the gear drive, the flapping frequency, and the flapping flexibility of the servos controlling the four wings are examined with flapping and without flapping. The results indicate that the transmission of the gear set is smooth with a flapping frequency range from $2 \mathrm{~Hz}$ to $8 \mathrm{~Hz}$, and servos can control the hinges to move neatly. The maximum and minimum lateral flapping angles of the prototype at $\tau= \pm 0.5$ are measured, as presented in Figure 9. At $\tau=0.5$, the maximum and minimum values of the left flapping angle are $55.2^{\circ}$ and $-38.0^{\circ}$, and those of the right flapping angle are $39.6^{\circ}$ and $-23.7^{\circ}$. These values are $39.6^{\circ}$ and $-22.1^{\circ}$ and $57.0^{\circ}$ and $-38.4^{\circ}$ at $\tau=-0.5$. These measured results are quite close to the calculated data shown in Table 2.

Finally, an outdoor experiment on turning flight is conducted to validate the feasibility of the SCSM with movable hinges. The prototype is powered by batteries of different weights, and it can effectively fly for 5 minutes. The prototype provides a load range from $10 \mathrm{~g}$ to $25 \mathrm{~g}$, which offers an important guarantee for carrying necessary equipment. A remote controller is used to control the movement of the hinges for the turning flight. The whole process of the turning test is completed smoothly and successfully, as shown by a series of pictures in Figure 10. The flight test shows that the proposed mechanism is perfectly capable of providing a controllable turning maneuver flight for the FMAV. Compared to FMAVs controlled by tails, the present prototype achieves turning flight by breaking the symmetry of the flapping angles of the lateral wings, which is more effective. It has been previously explained that dragonflies or insects turn by changing the positions of their lateral wings rather than their tails [22-24]. In addition, these experiments verify our previous numerical simulation [6]. This study enriches the knowledge of the existing flapping mechanism and provides a novel way to control the turning flight of an insect-sized FMAV with lateral wings rather than tails. Miniaturizing this prototype will be the focus of our future work.

\section{Conclusions}

Two flapping mechanisms with movable hinges based on the common flapping mechanisms, the DCRM and the SCSM, are proposed for studying the turning maneuverability for an FMAV. The movement of the hinges in these two mechanisms can cause the lateral flapping angles to be asymmetric to generate turning flights. A kinematics study of these two mechanisms is conducted, and it is found that the mechanism based on the SCSM has better performance than the mechanism based on DCRM. To see the feasibility of the mechanisms, a dragonfly-like FMAV with adjustable flapping amplitudes based on the SCSM is designed, and a prototype is developed. With this prototype, a test on an experimental platform and an outdoor flight test are carried out. The results show that the proposed flapping mechanism with movable hinges is perfectly capable of providing controllable turning during flight.

\section{Data Availability}

The authors are willing to share the data underlying the findings of this work, and the data used to support the findings of this study are included within the article.

\section{Conflicts of Interest}

The authors declare that there is no conflict of interest regarding the publication of this paper.

\section{Authors' Contributions}

Rui Zhang contributed to the conceptualization, modelling and simulation, and calculations. Haiyang Zhang contributed 
to the modelling and assembling. Lichao Xu contributed to the assembling and flight testing. Peng Xie contributed to the writing and revision. Jing $\mathrm{Wu}$ contributed to the conceptualization and revision. Chao Wang contributed to the discussions and writing.

\section{Acknowledgments}

This work was funded by the National Natural Science Foundation of China (52005104), the KEY Laboratory of Robotics and Intelligent Equipment of Guangdong Regular Institutions of Higher Education (2017KSYS009), the Shenzhen Fundamental Research Program (JCYJ20180306171753070), the Featured Innovation Projects in Colleges and Universities in Guangdong Province (2018KTSCX353; 2020KTSCX149), the Technology Project of Jiangsu Province of China (SZHA2019001), the Science and technology planning project of Huai' an (HAB202152), the Undergraduate Innovation and Entrepreneurship Training Program of Jiangsu Province (202111049008Z), the DGUT innovation center of robotics and intelligent equipment (KCYCXPT2017006), and the Research Start-up Funds of DGUT (211135025).

\section{References}

[1] D. Xue, B. Song, W. Song, and W. Yang, "Advance in coupling aeroelasticity and flight dynamics of birdinspired FMAV," Acta Aerodynamica Sinica, vol. 36, no. 1, pp. 88-97, 2018.

[2] L. Wang and F. Tian, "Numerical study of flexible flapping wings with an immersed boundary method: fluid-structureacoustics interaction," Journal of Fluids and Structures, vol. 90, pp. 396-409, 2019.

[3] D. E. Alexander, "Wind tunnel studies of turns by flying dragonflies," Journal of Experimental Biology, vol. 122, no. 1, pp. 81-98, 1986.

[4] D. E. Alexander, "Unusual phase relationships between the forewings and hindwings in flying dragonflies," Journal of Experimental Biology, vol. 109, no. 1, pp. 379-383, 1984.

[5] H. Wang, L. Zeng, H. Liu, and C. Yin, "Measuring wing kinematics, flight trajectory and body attitude during forward flight and turning maneuvers in dragonflies," Journal of Experimental Biology, vol. 206, no. 4, pp. 745-757, 2003.

[6] R. Zhang, C. Zhou, C. Wang, and P. Xie, "Aerodynamic characteristics of dragonfly in asymmetric flapping," Acta Aeronautica et Astronautica Ainica, vol. 38, no. 12, pp. 101-113, 2017.

[7] S. N. Fry, R. Sayaman, and M. H. Dickinson, "The aerodynamics of free-flight maneuvers in Drosophila," Science, vol. 300, no. 5618, pp. 495-498, 2003.

[8] R. Ramamurti and W. C. Sandberg, "A computational investigation of the three-dimensional unsteady aerodynamics of Drosophila hovering and maneuvering," Journal of Experimental Biology, vol. 210, no. 5, pp. 881-896, 2007.

[9] L. Ristroph, G. J. Berman, A. J. Bergou, Z. J. Wang, and I. Cohen, "Automated hull reconstruction motion tracking (HRMT) applied to sideways maneuvers of free-flying insects," Journal of Experimental Biology, vol. 212, no. 9, pp. 1324-1335, 2009.

[10] S. Vogel, "Flight in Drosophila," Journal of Experimental Biology, vol. 46, no. 2, pp. 383-392, 1967.
[11] F. T. Muijres, M. J. Elzinga, J. M. Melis, and M. H. Dickinson, "Flies evade looming targets by executing rapid visually directed banked turns," Science, vol. 344 , no. 6180, pp. 172177, 2014.

[12] X. Meng and M. Sun, "Aerodynamics and vortical structures in hovering fruitflies,” Physics of Fluids, vol. 27, no. 3, p. 031901, 2015.

[13] Y. Nan, B. Peng, Y. Chen, and D. McGlinchey, "From studying real hummingbirds to designing hummingbird-like robots-a literature review," IEEE Access, vol. 7, pp. 131785-131804, 2019.

[14] A. Roshanbin, F. Abad, and P. André, "Kinematic and aerodynamic enhancement of a robotic hummingbird," AIAA Journal, vol. 57, no. 11, pp. 4599-4607, 2019.

[15] F. Fei, Z. Tu, J. Zhang, and X. Deng, "Learning extreme hummingbird maneuvers on flapping wing robots," in 2019 International Conference on Robotics and Automation (ICRA), pp. 109-115, Montreal, QC, Canada, 2019.

[16] S. Timmermans, F. Leys, and D. V. Vandepitte, "Model-based evaluation of control roll, pitch, yaw moments for a robotic hummingbird," Journal of Guidance, Control, and Dynamics, vol. 40, no. 11, pp. 2934-2940, 2017.

[17] M. Jankauski, T. L. Daniel, and I. Shen, "Asymmetries in wing inertial and aerodynamic torques contribute to steering in flying insects," Bioinspiration \& Biomimetics, vol. 12, no. 4, p. 046001, 2017.

[18] G. K. Taylor, "Mechanics and aerodynamics of insect flight control," Biological Reviews, vol. 76, no. 4, pp. 449-471, 2001.

[19] U. Horsmann, H. G. Heinzel, and G. Wendler, "The phasic influence of self-generated air current modulations on the locust flight motor," Journal of Comparative Physiology, vol. 150, no. 4, pp. 427-438, 1983.

[20] F. O. Lehmann and M. H. Dickinson, "The control of wing kinematics and flight forces in fruit flies (Drosophila spp.)," Journal of Experimental Biology, vol. 201, no. 3, pp. 385-401, 1998.

[21] B. Cheng, S. N. Fry, Q. Huang, and X. Deng, “Aerodynamic damping during rapid flight maneuvers in the fruit fly Drosophila," Journal of Experimental Biology, vol. 213, no. 4, pp. 602-612, 2010.

[22] H. Liu, S. Ravi, D. Kolomenskiy, and H. Tanaka, "Biomechanics and biomimetics in insect-inspired flight systems," Philosophical Transactions of the Royal Society, B: Biological Sciences, vol. 371, no. 1704, p. 20150390, 2016.

[23] H. V. Phan, T. Kang, and H. C. Park, "Design and stable flight of a $21 \mathrm{~g}$ insect-like tailless flapping wing micro air vehicle with angular rates feedback control," Bioinspiration \& Biomimetics, vol. 12, no. 3, p. 036006, 2017.

[24] Q. V. Nguyen and W. Chan, "Development and flight performance of a biological-inspired tailless flapping wing micro air vehicle with wing stroke plane modulation," Bioinspiration \& Biomimetics, vol. 14, no. 1, p. 016015, 2019.

[25] M. Keennon, K. Klingebiel, and H. Won, "Development of the nano hummingbird: a tailless flapping wing micro air vehicle," in 50th AIAA Aerospace Sciences Meeting Including the New Horizons Forum and Aerospace Exposition, pp. 8634-8657, Nashville, Tennessee, 2012.

[26] N. Gaissert, R. Mugrauer, G. Mugrauer, A. Jebens, K. Jebens, and E. M. Knubben, "Inventing a micro aerial vehicle inspired by the mechanics of dragonfly flight," in Towards Autonomous Robotic Systems, A. Natraj, S. Cameron, C. Melhuish, and M. 
Witkowski, Eds., vol. 8069 of Lecture Notes in Computer Science, pp. 90-100, Springer, Berlin, Heidelberg, 2014.

[27] W. Duan, H. Ang, and T. Xiao, "Design and tunnel test of an ornithopter with differential twist wings," Journal of Experiments in Fluid Mechanics, vol. 3, pp. 37-42, 2013.

[28] S. Sunada, H. Wang, L. Zeng, and K. Kawachi, "Analysis of maneuvering flight of an insect," Journal of Bionic Engineering, vol. 1, no. 2, pp. 88-101, 2004.

[29] Y. Chen, M. Skote, Y. Zhao, and W. M. Huang, "Dragonfly (Sympetrum flaveolum) flight: kinematic measurement and modelling," Journal of Fluids and Structures, vol. 40, pp. 115126, 2013.

[30] Y. Tao, J. Zhou, X. Zhu, and L. X. Wu, "Discussion on the design of flapping-wing actuators," Machine Design and Research, vol. 22, no. 2, pp. 29-32, 2006. 